\title{
Le meilleur des deux mondes : combiner le présentiel et l'eLearning (microscopie virtuelle) dans des TPs d'histologie générale
}

\author{
Sandra Florquin ${ }^{(a)}$, Sylvie Multon ${ }^{(a)}$, Alodie Weatherspoon ${ }^{(a)}$, France Mélot ${ }^{(b)}$, Ingrid Dupierreux $^{(b)}$, \\ Patrick Schaffer $^{(\mathrm{c})}$, Pascale Quatresooz ${ }^{(\mathrm{a})}$, Valérie Defaweux ${ }^{(\mathrm{a})}$ \\ (a) Service d'Histologie Humaine, Faculté de Médecine, Université de Liège (Belgique) \\ (b) Bureau pédagogique de la Faculté de Médecine, Université de Liège (Belgique)

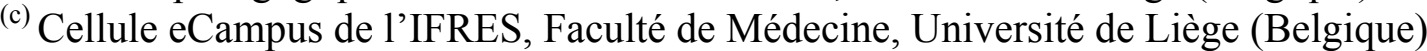

Depuis 2012, la réforme des études de Médecine en Belgique a entrainé le déplacement des travaux pratiques (TP) d'Histologie Générale jadis enseignés en deuxième année à la première année d'étude. Ce changement a donné lieu à une révision radicale de l'organisation des enseignements, en vue d'affronter les nouvelles contraintes - triplement du nombre d'étudiants, locaux sur-occupés, augmentation non proportionnelle des formateurs, microscopes et lames en nombre insuffisant - et d'accroitre pour tous la qualité de la formation.

Les lames histologiques, ressource d'apprentissage clé des disciplines telles que l'Histologie et l'Anatomie Pathologique, ont donné lieu au développement d'un dispositif d'auto-apprentissage en ligne, accessible aux 500 étudiants. Mis au point sur base d'expériences antérieures $[1,2,3]$, il invite les étudiants à parcourir, pour chaque famille de tissus, plusieurs lames présentant chacune des structures histologiques numérotées. Des annotations, commentaires, dessins ou photos sont associés à ces repères qui enrichissent l'étude des coupes, de la vue d'ensemble au niveau cellulaire. Ce «jeu de piste» donne à l'approche de la matière un caractère à la fois systématique et ludique, autonome et balisé. Des vidéos intégrées à l'outil en ligne complètent le dispositif en ligne.

Le volet d'auto-apprentissage est rigoureusement articulé à des «séances de clôture » délivrées en présentiel qui visent le renforcement des connaissances des étudiants, la vérification de leurs acquis et le transfert de ceux-ci à des situations nouvelles. Ce dispositif hybride $[4,5]$ allie les bénéfices d'un enseignement à distance [6] (personnalisation de l'apprentissage, accès démultiplié aux ressources) et en face-à-face [7] (interaction directe avec les personnes ressources). Une recherche actuellement en cours, combinant dans une méthodologie mixte l'analyse du feedback des étudiants et l'étude des traces d'apprentissage, permettra d'en cerner les effets sur diverses composantes de l'expérience d'apprentissage.

Mots clés : Travaux pratiques, Histologie humaine, e-Learning, innovation pédagogique Références bibliographiques :

[1] Helle L, Nivala M, Kronqvist P. More Technology, Better Learning Resources, Better Learning? Lessons from Adopting Virtual Microscopy in Undergraduate Medical Education. Anat Sci Educ 2013;6:73-80

[2] Triola et Holloway. Enhanced virtual microscopy for collaborative education. BMC Medical Education 2011;11:4

[3] Collier L, Dunham S, Braun MW, O'Loughlin VD. Optical versus virtual: Teaching assistant perceptions of the use of virtual microscopy in an undergraduate human anatomy course. Anat Sci Educ 2012;5:10-19 
[4] Pereira JA, Pleguezuelos E, Merí A, Molina-Ros A, Molina-Tomás MC, Masdeu C. Effectiveness of using blended learning strategies for teaching and learning human anatomy. Medical Education 2007;41(2):189-195

[5] Elen J. Het hoger onderwijs zal blended zijn of niet zijn [L'enseignement supérieur sera hybride ou ne sera pas] 2011. Exposé d'ouverture à la conférence du Conseil de l’Enseignement Supérieur (Vlhora, Bruxelles). 